\title{
The Creativity Matrix: An Extension To Axiomatic Design
}

\author{
Brad Crowell \\ Department of Electrical \\ \& Computer Engineering \\ Dalhousie University \\ Halifax, N.S. \\ bdcrowell@hfx.eastlink.ca
}

\author{
Dr. Peter Gregson \\ Department of Electrical \\ \& Computer Engineering \\ Dalhousie University \\ Halifax, N.S. \\ Peter.Gregson@Dal.Ca
}

\begin{abstract}
Axiomatic Design helps a designer to make good design decisions. However, this addresses only one part of design. Prior to selecting a proposed design, the designer must synthesize options for further consideration. Within engineering design, creativity and expertise have been left to the competency of the designer and called the "art of engineering design".

To achieve a truly creative design process that addresses both analysis and synthesis, methods based on theories from Cognitive Psychology must be included. The resulting Creative Axiomatic Design process addresses both synthesis and analysis, enhancing creativity and expertise to inspire innovation and alternative perspectives on the design problem.
\end{abstract}

\section{Introduction}

The design axioms put forth in Axiomatic Design provide the basis for the analysis tools that are especially useful during the early, conceptual design stage of design [1]. This early stage of design is also important when considering creativity as it offers the best opportunity to inject innovation into a design solution [2]. Unfortunately, Axiomatic Design does not explicitly address creativity within its present framework.
There has been much research on creativity and expertise within the science of Cognitive Psychology, but little of this work has penetrated into the engineering design process. Within engineering design, creativity and expertise have been left to the competence of the individual designer and labelled as the "art of engineering design". Until an engineering design process addresses both the traditional analysis aspects of design and the synthesis processes that embody creativity, the "art" of engineering design will continue to exist. As long as engineering design is considered to be an "art," it will not be taught as a controllable, manageable and effective process and so its development will suffer.

Cognitive Psychology has shown that knowledge of the basic principles of the problem domain and its interconnection with practical applications are the crucial differences in the problem-solving performance of experts and novices. Ensuring that a designer is approaching a problem using the basic principles of the knowledge domain is critical for the effective synthesis of potential solutions.

It is important to recognize that design analysis, in isolation, does not enhance the designer's ability to provide creative solutions. It is "the interaction of synthesis and analysis [that] leads to design creativity" [3, p. 2]. The domain knowledge of the designer directly affects the quality of the solution developed. However, the approach used to exploit this knowledge has a larger effect on the creativity of the solution [3]. 
To address these issues, an extension to the Axiomatic Design framework is proposed. Thus, by explicitly integrating a methodology and supporting design tool to enhance creativity and expertise into this design process, an extended Axiomatic Design process can address both the synthesis and analysis activities required in engineering design. This new "Creative Axiomatic Design" should result in better design solutions as the enhancement of creativity and expertise inspires innovation and alternative perspectives on the design problem.

\section{Background}

\subsection{Axiomatic Design}

According to Suh, Axiomatic Design offers a "scientific treatment" [4, p. 2] of the design process. Axiomatic Design provides a framework and a set of axioms that can be applied to any design problem; engineering, business, computer software, etc.

The Axiomatic Design framework defines four domains that differentiate the main activities included within the design process. In the Customer Domain, the designer is focused on defining the customer attributes (CAs) of the problem that are of concern to the customer. In the Functional Domain, those CAs are used to define a solution-neutral, independent set of functional requirements (FRs). The design parameters (DPs) in the Physical Domain are identified to satisfy those requirements. Finally, the Process Domain is focused on the production of those DPs through the identification of process variables (PVs) [4].

The mapping between the domains in Axiomatic Design is defined by design matrices. The first matrix defines the relationship between the FRs in the Functional Domain and the DPs in the Physical Domain. A second Design Matrix maps the physical DPs to the PVs in the Process Domain. This provides two design equations that describe the system architecture for a given design [4].

The designer's creativity during the design process usually results in many alternatives that fulfill the FRs and therefore the CAs. Axioms are the basis for design decisions in Axiomatic Design and are used to choose the best of these alternatives. [4].

The Independence Axiom helps the designer analyze the proposed designs and decide which are worthy of further consideration. The Independence Axiom requires that the FRs for a design must remain independent of one another. The list of FRs is "the minimum set of independent requirements that characterize the design goals" [4, p. 16]. If multiple designs satisfy the Independence Axiom, Suh's Information Axiom is applied. It states that the design solution with the least information content is the best one [4].

A design is characterized by its FRs and constraints. Within the list of constraints, Sohlenius, Fagerstrom and Kjellberg proposed that two are always present; energy and time. They argue that minimizing the amount of time and energy required to design and produce a designed object is an underlying requirement for every design. By applying these goals as axioms, the design process minimizes them while providing a design that meets the Independence and Information Axioms. [5].

While addressing the analysis process in design, the Axiomatic Design framework only indirectly addresses the creative aspects of the design process. It relies on the individual capabilities of the designer to synthesize the designs that can subsequently be analyzed.

\subsection{Genplore}

Cognitive psychology is the study of thinking in its most general terms. Cognition includes "the interpretation, manipulation, and transformation of mental representations" [6]. Creative cognition is a field within the broader study of Cognitive Psychology specifically concerned with our understanding of creativity [7]. The Genplore theory has been proposed to explain the cognitive processes of creative thought [8]. Genplore gets it name from the two main aspects of cognitive processes, generative and exploratory, that it models [8]. 
Benami and Jin applied the Genplore model to conceptual design because it is during this phase that the designer has the freedom to generate multiple solutions to the design problem. These early design ideas have a significant effect on the eventual cost and quality of the final design [9].

To address the cognitive aspects of conceptual design, they proposed the Cognitive Model of Creative Conceptual Design in which a design object or artefact is comprised of components called design entities. Design entities have three features; function, form, and behaviour. The function of a design entity is the operation required to satisfy the design requirements. The form of the entity is the physical shape and structure. The means used to provide the function is its behaviour [9].

Benami and Jin identified the main patterns of stimulation, production, and generation that occur during conceptual design that tend to be the most creative. The main cognitive processes of Memory Retrieval, Problem Analysis, and Solution Analysis tend to produce suggestions, declarations, and explanations. These design operations generate a design entity that fulfills the design requirements to some extent. The behaviour and form of that entity, more so than its function, stimulate further cognitive processes [9]. By focusing on these more abstract aspects of the design entity, specifically its behaviour and form, the designer can develop more innovative designs [7]. Since Axiomatic Design focuses on the function of a design entity, DP or PV, this point is of particular significance.

Genplore provides the theoretical basis for the Cognitive Model of Creative Conceptual Design, which identified the main patterns of stimulation, production, and generation that occur during conceptual design. Benami and Jin show that the behaviour and form of a design entity stimulate creativity more than its function [9], resulting in more innovative designs [7].

\subsection{Mental Representations}

It is known that both quantitative and qualitative knowledge are necessary for problem solving and engineering design [10] Even partial qualitative knowledge significantly increases problem-solving ability [10]. Use of both qualitative and quantitative knowledge is a major difference between the mental models of expert and novice problem solvers with respect to [11]:

1. sophistication,

2. completeness, and

3. appropriateness.

Experts' mental models are more sophisticated by using extensive domain knowledge and linking quantitative and qualitative knowledge. They seek a complete mental representation before attempting to solve the problem. Qualitative knowledge is used to refine the mental model and ensure its appropriateness [11].

The common themes that become evident when addressing expertise are 1) mastery of the basic principles and 2) connections between basic principles and professional practice within the domain. The quantity and quality of fundamental domain knowledge directly affects the sophistication, completeness, and appropriateness of the resulting mental representations. Thus, obtaining this foundation is vital in design engineering. The links between these basic principles and their practical application are equally important [11].

\section{Creative Axiomatic Design}

Axiomatic Design enhances analysis through Design Matrices and axioms to avoid implementing an inferior solution [4], but lacks specific tools and techniques for synthesis. In contrast, the Cognitive Model of Creative Conceptual Design shows that creativity is enhanced when the designer focuses on designentity behaviour and form [9].

Cognitive Psychology has also studied expertise, revealing that mastery of knowledge-domain basic principles and making connections between them and professional practice are critical to expertise. Design problems must be addressed from basic principles to ensure 
mental models that are sophisticated, complete, and appropriate.

To achieve a truly creative design process that addresses both analysis and synthesis, the relevant theories from Cognitive Psychology must be included in Axiomatic Design. Two issues must be addressed. The first is the modifications required to Axiomatic Design to incorporate the creative aspects of design. The second issue requires making creativity explicit within Axiomatic Design. A design tool is required that focuses the designer on creativity in the design process to enhance their creative abilities.

\subsection{Creativity in the Design Process}

Incorporating creativity explicitly in Axiomatic Design is not simple. A new definition of the design process is required. This results in a significantly different design process and so the flow through this new process is also different.

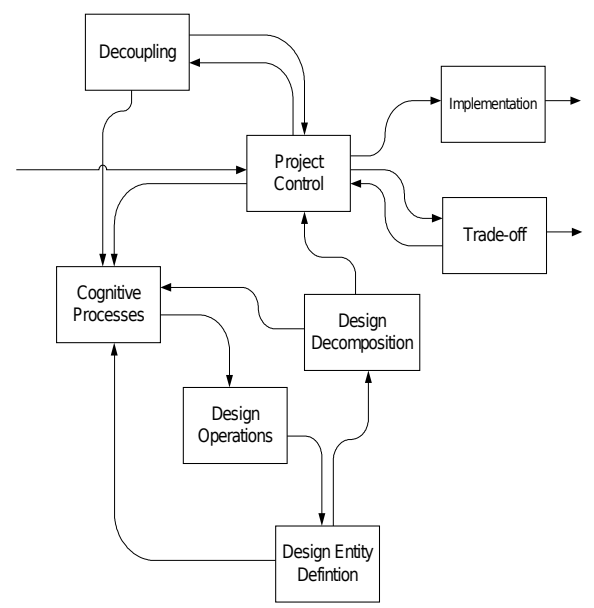

Figure 1 Creative Axiomatic Design

\section{Process}

- Project Control - the starting point, defines the initial scope and the planning and control of the project as it progresses.

- Design Decomposition - primarily analysis. Design Matrix is developed and associated technical decisions made, including selection of the desired solution candidate.

- Cognitive Processes - first step in the creative loop, including generative processes (Memory Retrieval, Associations, and Transformations) and exploratory processes (Problem and Solution Analysis) produce design operations.

- Design Operations - second creative loop step. Internal, external design operations generate design entities. - Design Entity Definition - final step in the creative loop.

- Focuses on behaviour and form of a design entity to stimulate further Cognitive Processes resulting in additional iterations around this loop.

- Decoupling - an analysis step, includes decoupling of a coupled design.

- Implementation - an exit step, setting of DP and PV values for chosen design solution.

- Trade-off - an exit step, maximizing performance of a coupled design; or trade-off process may be unsuccessful, designer goes back to Project Control to further assess the proposed design.

\subsection{A Framework for Creativity}

The second issue is how to include creativity as an explicit activity within Creative Axiomatic Design. Maximizing designers' creativity requires a design tool that: 1) focusses designers on behaviour and form of the design entities [9] and 2) ensures that designers' mental models are sufficiently appropriate, complete, and sophisticated for the problem [11].

An additional matrix is proposed to explicitly enhance the designer's creativity within Creative Axiomatic Design. For a particular FR, this Creativity Matrix maps the proposed DPs to their underlying behaviours (Equation 1). Also, for a particular DP, another Creativity Matrix can map the proposed PVs to their underlying behaviours (Equation 2). A Creativity Matrix can be instantiated for each FR and DP identified in the design hierarchy if desired.

$$
\begin{array}{ll}
{[D P]=[C][b]} & \text { Equation 1 } \\
{[P V]=[D][b]} & \text { Equation 2 }
\end{array}
$$

where: $\mathrm{b}$ is the behaviour of the DP or PV. 
The matrix can be expanded in tabular format, as shown in Table 1.

Table 1 Creativity Matrix for $F R_{x}$

\begin{tabular}{l|c|c|c} 
FR $_{\mathbf{x}}:$ & \multicolumn{1}{|c}{} \\
& $\mathbf{b}_{\mathbf{x a}}$ & $\mathbf{b}_{\mathbf{x b}}$ & $\mathbf{b}_{\mathbf{x c}}$ \\
\hline DP $_{\mathbf{x a}}$ & $\mathrm{X}$ & & \\
\hline $\mathbf{D P}_{\mathbf{x b}}$ & & $\mathrm{X}$ & \\
\hline $\mathbf{D P}_{\mathbf{x c}}$ & & & $\mathrm{X}$
\end{tabular}

Assembling the Creativity Matrix focuses the designer on DP or PV behaviour rather than function. This avoids functional fixation. Subsequent steps use design-entity form to stimulate Cognitive Processes and, potentially, additional solutions.

When a designer considers how to achieve a particular FR, one or more cognitive processes result in an initial DP. If that DP is satisfactory, no further design is required. Otherwise, the DP can be mapped to its behaviour using the Creativity Matrix. This will stimulate cognitive processes that produce design operations that in turn generate additional behaviours or DPs. These newly identified DPs will also fulfill the original FR. By focusing the designer on the behaviour of the DPs, the Creativity Matrix maximizes the potential for creative solutions.

The Creativity Matrix modifies the "function before form" mantra common in engineering design. Design-entity function is still the key goal, but behaviour and form are used to stimulate creativity with function used to keep the process on track to a solution. The design mantra could be stated as "form follows behaviour to achieve function" for Creative Axiomatic Design.

As additional behaviours and their DPs are generated, the Creativity Matrix is expanded. It can take various forms, diagonal, triangular, or full. We contend that the Creativity Matrix must be diagonal to ensure the use of basic principles, a highly restrictive condition. Any form other than a square matrix with diagonal elements reveals an inconsistent mental model with respect to the basic principles of the knowledge domain.

It is intuitively apparent that multiple row mappings should not be possible if the designer is using basic principles to develop a mental model. For this to occur, a single DP must use more than one behaviour. When considering a design-entity behaviour in terms of basic principles, only one principle should apply. In this case, the mental model is either 1) not sophisticated and/or complete enough to properly address the design problem; or 2) it is fundamentally flawed and is therefore not appropriate for the design problem. In either case, the Creativity Matrix triggered redefinition of the designer's mental model.

A Creativity Matrix with multiple relationships in a column requires that different DPs map to a single behaviour, implying that an identified behaviour actually has multiple behaviours or that one of the two DPs is redundant. The matrix again triggers rethinking the problem in terms of basic principles.

This leads to a fifth axiom for Creative Axiomatic Design:

Axiom 5: The association of DPs and PVs to their underlying behaviours must ensure that each DP and PV is mapped to a single, unique and independent behaviour to be consistent with the basic principles of the knowledge domain.

\subsection{Assessing Design Entity Form}

The Creativity Matrix provides a list of potential DPs and the behaviours they each use to satisfy the FR. However, it is not necessarily complete. When considering which option would best suit the design problem, the form of the design entity must now be considered. A strictly behavioural DP description cannot fulfill a FR without identification of its form. Now the desired behaviour is assessed and the possible forms a design entity could take to embody this behaviour are identified.

Multiple forms might be possible for a design entity. The identification of one form may lead to the identification of another through the same cognitive processes used to generate and explore the behaviours. Furthermore, a form may be explored by the designer and altered in such a way as to generate an additional behaviour. This behaviour could then 
identify a DP that hadn't yet been considered. Thus, the creative process continues after the Creativity Matrix has been assembled, through the process of DP and behaviour identification and restatement. These Creativity Matrix restatements ensure the consistency of the designer's mental model with the basic principles.

When the designer has the required amount of information on each design option under consideration, the DP and its form are chosen to best fulfill the associated FR.

\section{Conclusions}

When attempting to solve a design problem, the designer may not have the needed information or may have an incorrect depiction of the problem [12]. The Creativity Matrix assists with both of these problems. First, by focusing the designer on the basic principles of the domain, the Creativity Matrix helps to make assumptions explicit and allows the designer to address the completeness and sophistication of their mental model. Second, inconsistencies in the designer's mental model are identified, triggering reassessment of the mental model.

By explicitly integrating a design methodology and tool for creativity into its design process, Creative Axiomatic Design addresses synthesis and analysis activities required in engineering design and results in better design solutions.

\section{References}

1) Harutunian Vigain, Mats Nordlund, Derrick Tate, and Nam P. Suh, "Decision Making and Software Tools for Product Development Based on Axiomatic Design Theory," in Proc. CIPR Annals, Vol.45/1, 1996, pp. 135-140.

2) Dr. David H Cropley, and Arthur J Cropley, "Creativity and Innovation in the Systems Engineering Process," in Proc. INCOSE, 2000, pp. 807-814;

3) Yong Zeng, Baiquan Yan, Bo Chen, and Shengii Yao, "A Theoretical and Experimental Study on Design Creativity" presented at the Inaugural CDEN Design Conference, Montreal, Canada, 2004.

4) Nam Pyo Suh, Axiomatic Design: Advances and Applications, New York, NY, Oxford University Press, 2001.

5) Gunnar Sohlenius, Jonas Fagerstrom, Ann Kjellberg, "The Innovation Process and the Principal Importance of Axiomatic Design," in Proc. ICAD, 2002, 018 pp.1-8.

6) Robert J.Sternberg, Thinking and Problem Solving: Handbook of Perception and Cognition, San Diego, CA, Academic Press, 1994.

7) Thomas Ward, Steven Smith, and Ronald Finke, "Creative Cognition," in Handbook of Creativity, Robert Sternberg Ed. Cambridge, UK, Cambridge University Press; 1999, pp. 189-212.

8) Robert Finke, Thomas Ward, and Steven Smith, Creative Cognition, Cambridge Mass, The MIT Press, 1992.

9) Oren Benami and Yan Jin, "Creative Stimulation in Conceptual Design," in Proc. ASME DETC, 2002, DTM-23023 pp. 1-13.

10) Rolf Ploetzner and Hans Spada, "Multiple Mental Representations of Information in Physics Problem Solving," in The Cognitive Psychology of Knowledge, Gerhard Strube, Karl F.Wender, Ed.

11) Brad Crowell and Peter Gregson, "The Importance of Mental Representations in Design Engineering" in Proc. CDEN, 2004

12) Thomas T. Hewett, Cognitive Factors in Design: Overview and Some Implications for Design, in Proc. Creativity and Cognition, 2005, pp. 318 321. North-Holland, Elsevier Science Publishers, 1993, pp. 289-309. 